\title{
Descriptive Analysis Of Clinical Presentation And Manage- ment Of Pancreatic Trauma At A Level I Trauma Centre
}

\author{
Hassan Al-Thani MD1', Ahmed Faidh Ramzee MD² , Ammar Al-Hassani MD', Gustav Strandvik MD', Ayman \\ El-Menyar,MD 3,4
}

1Trauma Surgery Section, Hamad General Hospital (HGH), Doha, Qatar (althanih@hotmail.com), ( gstrandvik@hamad.qa), (ammar_alhassani@yahoo.com)

2 Department of Surgery, Hamad General Hospital (HGH), Doha, Qatar (aabdulaziz1@hamad.qa)

3 Clinical Research, Trauma \& Vascular Surgery, Hamad General Hospital, Doha, Qatar 4 Clinical Medicine, Weill Cornell Medical College, Doha, Qatar

(aymanco65@yahoo.com)

* Correspondence

Ayman El-Menyar, MD

Trauma \& Vascular Surgery Section, Hamad Medical Corporation \& Weill Cornell Medical College, PO Box 3050, Doha, Qatar

Tel: 0097444396130

E-mail: aymanco65@yahoo.com

Abstract: Background: We aimed to study the frequency, management, and outcomes of patients with blunt pancreatic trauma.

Methods: We reviewed the medical records for all patients admitted with pancreatic injuries between 2011 and 2017 at the only level 1 trauma center in the country.

Results: There were 71 patients admitted with pancreatic trauma $(0.6 \%$ of trauma admissions and $3.4 \%$ of abdominal injury admissions) with a mean age of 31 years. Sixty-two patients had pancreatic injury grade I-II and 9 had grade III-IV. Thirty-eight percent had GCS $<9$ and $73 \%$ had ISS $>16$. The level of pancreatic enzymes was significantly proportional to the grade of injury. Over half of patients required a laparotomy, of them 12 patients had an intervention on the pancreas. Eight patients developed complications related to pancreatic injuries ranging from pancreatitis to pancreati-

co-cutaneous fistula while 35\% developed hemorrhagic shock. Mortality was 31\% and regardless of the grade of injury, the mortality was associated with high ISS, low GCS and presence of hemorrhagic shock.

Conclusion: Pancreatic injuries following blunt trauma are rare and the injured subjects are usually young male. However, most injuries are of low-grade severity. This study shows that shock, higher ISS and lower GCS are associated with worse in-hospital outcomes. Non-operative management may suffice in patients with lower grade injuries, which may not be the case in patients with higher grade injuries unless carefully selected.

Keywords: trauma, pancreas, abdominal injury, head injury, polytrauma

\section{Introduction}


Pancreatic injury following abdominal trauma is a rare entity, however it is usually associated with other visceral injuries and entails significant morbidity and mortality. The morbidity and mortality rates vary as $23.4-53 \%$ and $17.5-70 \%$ respectively $(1-3)$.

Integrity of the pancreatic duct is the most important factor for appropriate decision making because it determines the morbidity and mortality after pancreatic injury (4). Pancreatic injuries often constitute a major diagnostic and therapeutic challenge. A high index of suspicion is necessary as the retroperitoneal location of the pancreas contributes to a delay in the presentation of symptoms, signs, and biochemical changes in the initial stages of injury $(3,5)$. The incidence of pancreatic injury has been reported in $0.4 \%-3.6 \%$ of all trauma admissions $(1,6)$ and $3.7-11 \%$ in patients with abdominal trauma $(7-10)$. Blunt trauma accounting for most of cases (61-85\%) as opposed to penetrating trauma. There is an epidemiological variation worldwide with relation to the mechanism of injury (blunt vs penetrating) owing to social and cultural differences; with most regions reporting a higher number of blunt injury in contrary to regions where easy access to firearms result in a larger cohort of penetrating injuries as reported in South Africa and USA (2).

Initial diagnosis of pancreatic injuries may not be straightforward as clinical as well as radiological signs may be initially subtle, and this may contribute to a delay in diagnosis and therefore increased morbidity and mortality. Contrast-enhanced CT-scan is the fastest and most comprehensive technique for detecting suspected pancreatic injuries and is the modality of choice in hemodynamically stable patients. Contrast-enhanced CT-scan has high specificity (90-95\%) but low sensitivity (52-54\%) for ductal involvement. Up to $40 \%$ of pancreatic injuries can be missed or misdiagnosed (11-13). Pancreatic injuries become more evident 12-24 h after trauma (14).

Serum amylase levels are neither sensitive nor specific for definitive screening or diagnosis of pancreatic injuries, particularly within 3-6 h after injury. Serum lipase is more specific than amylase; serum lipase may help screening of patients with clinical suspicion of pancreatic injuries (15). Amylase is normal at admission in up to $40 \%$ of patients with pancreatic trauma, and elevated levels are not specific for pancreatic trauma. Decreasing enzyme levels have been correlated with predicting success of non-operative management (NOM) (16-18). Accurate identification of signs of pancreatic trauma and its grading is mandatory to plan the best treatment strategy (6).

Many scoring systems are available for defining categories of pancreatic injuries however the most widely accepted grading system is the Organ Injury Scaling (OIS) developed by the American Association for the Surgery of Trauma (AAST). With this system, typically, higher-grade injuries correlate with higher mortality and complications (19). Recently, the World Society of Emergency Surgery and the AAST expert panel released guidelines for the management of duodenal, pancreatic and extrahepatic biliary tree trauma and provided a classification system which combines the AAST-OIS classification with the hemodynamic status of patients (9).

Operative management of pancreatic injuries depends on the grade of injury as well as associated injuries and can range from simple drainage for minor injuries to distal pancreatectomy and more complex reconstructive procedures and pancreatico-duodenectomy for extensive injuries (11).

Many studies showed that NOM for pancreatic trauma may be safe and effective in selected patients. Selection of patients for NOM is the key. It is widely acceptable that if the patient is stable with a low-grade injury, in the absence of any associated injury mandating explorative laparotomy, NOM can be attempted first (12, 20-23).

The only level I trauma centre of Hamad Medical Corporation in Qatar serves a population of 2.6 million and receives an average of 1500 - 1800 trauma admissions annually. There is a lack of information of the pancreatic trauma in our region. We aim to review our institution's experience of pancreatic injuries in adult subjects in terms of frequency, management and outcomes.

\section{Materials and Methods}


A retrospective analysis of prospectively maintained data of all patients with pancreatic injuries post trauma managed at the only level I trauma tertiary facility in the country between 2011 and 2017 was conducted. Records of all patients with pancreatic injury were reviewed and data pertaining to the following were gathered; demographics, and mechanism of injury, Glasgow coma scale (GCS), hemodynamic parameters, amylase and lipase levels, associated injuries, Injury severity score (ISS), Abbreviated Injury score (AIS), pancreatic injury grade (AAST-OIS), imaging interventions (ultrasonography and CT scan), laboratory tests (serum amylase and lipase), length of stay , complications and management (NOM and surgical intervention). On arrival to the trauma room in the ED, all patients underwent thorough clinical assessment and resuscitation according to Advanced Trauma Life Support (ATLS) guidelines. Our routine trauma investigations include: plain chest and pelvic x-ray followed by pan-CT scan and later on we may consider follow up CT scan and or MRCP depending on the clinical evaluation and complexity of the injury, finally we may consider ERCP as diagnostic and therapeutic tool if indicated. The non-operative management (NOM) includes 1-initial Nil Per Os (NPO) with or without nasogastric tube insertion. 2- depends on the clinical assessment and biochemical markers, will start PO feeding with clear fluid and gradually, if tolerated, to proceed with full diet. 3-in some cases with evidence of pancreatitis, fat free diet will be started. 4-If no signs of improvement, further investigation with MRCP to rule out complications that may require intervention and to study the integrity of pancreatic duct anatomy 5- also, in some cases with pancreatic leak or severe pancreatitis, a short term octreotide therapy will be added. This study obtained ethical approval from Research Ethics Committee, at Medical Research Center, Hamad Medical Corporation (HMC), Doha, Qatar (IRB\#14409/14\& IRB \# MRC-01-18-003) with a waiver of consent.

Statistical analysis: Data were presented as proportion, median and range, or mean \pm standard deviation, as appropriate. Differences in categorical variables (Grade I,II,III vs VI) , (ISS $<16$ vs ISS $>16)$, (GCS $<9$ vs GCS $>9$ ), and hemorrhagic shock vs no hemorrhagic shock) were analyzed using Chi-Square test. Normality of continuous variables was checked by Kolmogorov-Smirnov test. Continuous variables were compared using Student's t-test for 2 groups or ANOVA test for $>2$ groups, for parametric data. Mann-Whitney U test and Kruskal Wallis test were used for non-parametric data, whenever applicable. A two-tailed P value of $<0.05$ was statistically significant. All data analyses were carried out using the Statistical Package for the Social Sciences, version 18 (SPSS, Inc., Chicago, IL).

\section{Results}

Our cohort included 71 patients with pancreatic injury ( $0.6 \%$ of total trauma admissions and 3.4\% of total abdominal injury admissions) of which $69(97.2 \%)$ were males. Patients' age ranged from 18.6-43 years (Mean 31). Most cases sustained polytrauma. Blunt injury was the most predominant type of trauma accounting for $91.5 \%$ of cases while only 6 patients had penetrating injuries. Among blunt trauma, motor vehicle collisions were the most common mechanism of injury followed by fall from height. Table 1shows patients' demographics, clinical presentation, associated injuries, procedures, complications and outcomes in patients with pancreatic injury. The mean abdominal AIS was $2.9 \pm 0.9$, pancreatic injury scale was $1.72 \pm 0.86$ and ISS score was $27.2 \pm 14.5$. Most patients had pancreatic contusions $(n=56)$ and location of injury was almost evenly distributed between the head, body and tail of the pancreas. Figure 1 shows illustration of pancreatic injury grades (I to V). Peripancreatic hematomas were present in $36.6 \%$ of patients. Associated extra abdominal injuries were mainly head and chest $(42 \%$ and $38 \%$ respectively) that was mostly seen with low grade pancreatic injuries. 


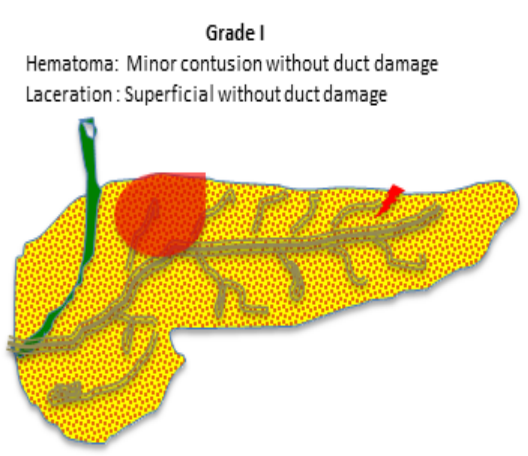

Grade III

Laceration: Distal division or parenchymal damage with ductal injury

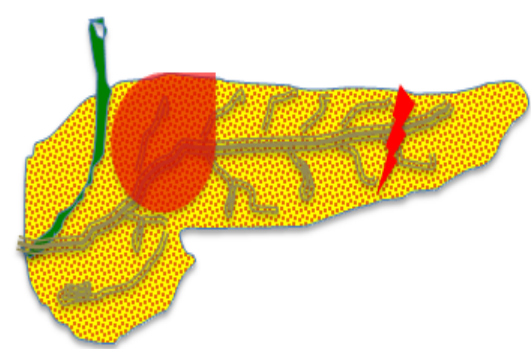

Grade V

Grade II

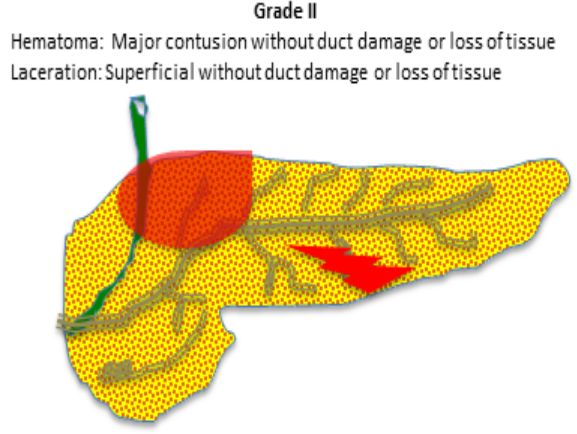

Grade VI

Laceration: Proximal division or parenchymal damage affecting the ampulla of Vater

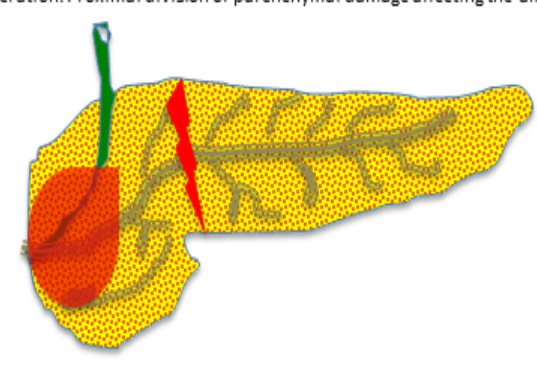

Laceration: Massive disruption of the head of the pancreas

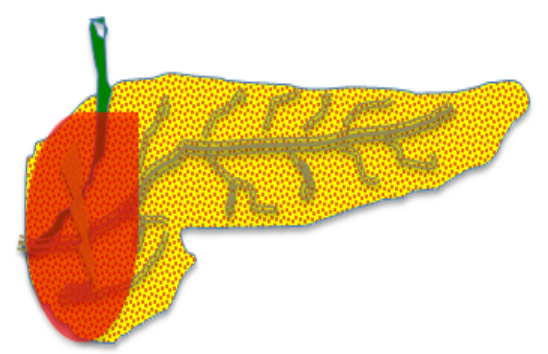

Figure 2: illustration of pancreatic injury grades

Biochemical marker levels (amylase and lipase) revealed average levels of $53 \mathrm{U} / 1$ and $84 \mathrm{U} / \mathrm{l}$ respectively but the degree of elevation was significantly proportional to the grade of pancreatic injury. Almost $17 \%$ patients were found to be under the influence of alcohol, with average ethanol levels at $35.6 \mathrm{mmol} / \mathrm{l}$.

Focused assessment with sonography in trauma (FAST scan) was positive in around half of patients and a CT scan of the abdomen was done in every case. Blood transfusions were required in 51 of the 71 patients with an average of 8 units transfused. Eight out of the 71 patients suffered from complications related to pancreatic injuries (i.e., collections, pancreatitis, pseudocyst and fistula). In-hospital complications (i.e., pneumonia, sepsis, organ failure and coagulopathy) are given in Table 1. 
Table 1: Demographics, clinical presentation, associated injuries, procedures, complications and outcomes in patients sustained pancreatic injury $(n=71)$

\begin{tabular}{|c|c|c|c|}
\hline Variable & Value & Variable & Value \\
\hline Age $($ mean \pm SD $)$ & $30.8 \pm 12.2$ & Pancreatic head & $25(35.2 \%)$ \\
\hline Males & $69(97.2 \%)$ & Pancreatic tail & $27(38.0 \%)$ \\
\hline Trauma type & & Pancreatic body & $22(31.0 \%)$ \\
\hline Blunt & $65(91.5 \%)$ & Peripancreatic hematoma & $26(36.6 \%)$ \\
\hline Penetrating & $6(8.5 \%)$ & Intubated ETT & $44(62.0 \%)$ \\
\hline GCS ED & $14(3-15)$ & CT scan abdomen & $71(100 \%)$ \\
\hline Serum lipase & $84(13-2057)$ & FAST (positive) & $34(47.9 \%)$ \\
\hline Serum amylase & $53(8-1107)$ & Procedures & \\
\hline Associated injuries & & Chest tube insertion & $36(50.7 \%)$ \\
\hline Head & $30(42.3 \%)$ & Exploratory laparotomy & $37(52.1 \%)$ \\
\hline Lung contusion & $24(33.8 \%)$ & Open reduction internal fixation & $12(16.9 \%)$ \\
\hline Pneumothorax & $20(28.2 \%)$ & Spinal surgery & $3(4.2 \%)$ \\
\hline Hemothorax & $13(18.3 \%)$ & Thoracotomy & $4(5.6 \%)$ \\
\hline Hemo-pneumothorax & $6(8.5 \%)$ & Pancreatic injury complications & $8(11.3 \%)$ \\
\hline Rib fracture & $27(38.0 \%)$ & Collection had CT-guided drainage & $4(50.0 \%)$ \\
\hline Spleen & $23(32.4 \%)$ & Pancreatitis & $2(25.0 \%)$ \\
\hline Liver & $21(29.6 \%)$ & Pseudocyst & $1(12.5 \%)$ \\
\hline Small Bowel & $8(11.3 \%)$ & Pancreatic cutaneous fistula & $1(12.5 \%)$ \\
\hline Mesentery & $11(15.5 \%)$ & Hospital length of stay & $14(1-61)$ \\
\hline Kidney & $11(15.5 \%)$ & ICU length of stay & $6(1-39)$ \\
\hline Stomach & $5(7.0 \%)$ & Ventilatory days & $8(1-30)$ \\
\hline Mesenteric vein & $3(4.2 \%)$ & Blood transfusion & $51(71.8 \%)$ \\
\hline Inferior vena cava & $1(1.4 \%)$ & Blood units transfused & $8(1-32)$ \\
\hline Aortic injury & $0(0.0 \%)$ & In-hospital complications & \\
\hline Diaphragmatic injury & $3(4.2 \%)$ & Wound Infection & $11(15.5 \%)$ \\
\hline Retroperitoneal hematoma & $18(25.4 \%)$ & Pneumonia & $11(15.5 \%)$ \\
\hline Injury severity & & Acute respiratory distress syndrome & $5(7.0 \%)$ \\
\hline Head AIS & $4.3 \pm 0.9$ & Sepsis & $9(12.7 \%)$ \\
\hline Chest AIS & $2.9 \pm 0.7$ & Acute renal failure & $3(4.2 \%)$ \\
\hline Abdomen AIS & $2.9 \pm 0.9$ & Coagulopathy & $1(1.4 \%)$ \\
\hline Pelvis AIS & $2.3 \pm 0.6$ & Mortality* & $22(31.0 \%)$ \\
\hline Injury Severity Score & $27.2 \pm 14.5$ & & \\
\hline Pancreatic laceration & $19(26.8 \%)$ & & \\
\hline Pancreatic contusion & $56(78.9 \%)$ & & \\
\hline $\begin{array}{l}\text { Prominent pancreatic } \\
\text { lobulation }\end{array}$ & $2(2.8 \%)$ & & \\
\hline Bulky pancreas & $1(1.4 \%)$ & & \\
\hline
\end{tabular}


* 20 head injury, 1 associated vascular injuries, 1 multiorgan failure

Table 2 shows demographics, clinical presentation and associated injuries by pancreatic injury scale according to the AAST pancreatic organ injury scale. Low grade pancreatic injuries were predominant, accounting for $87.3 \%$ of all patients (34 had Grade I and 28 had Grade II injuries) while 4 patients had Grade III and 5 had Grade IV injuries. There was no patient with Grade V pancreatic injuries in this cohort.

\begin{tabular}{|c|c|c|c|c|c|}
\hline & $\begin{array}{l}\text { Grade } \\
(n=34)\end{array}$ & Grade II $(n=28)$ & Grade III $(n=4)$ & Grade IV $(n=5)$ & P-value \\
\hline Age $($ mean \pm SD $)$ & $29.5 \pm 12.4$ & $34.5 \pm 11.7$ & $20.0 \pm 10.9$ & $28.8 \pm 8.2$ & 0.10 \\
\hline Males & $33(97.1 \%)$ & $27(96.4 \%)$ & $4(100 \%)$ & $5(100 \%)$ & 0.95 \\
\hline GCS ED & $14(3-15)$ & $14(3-15)$ & $15(15-15)$ & $15(3-15)$ & 0.19 \\
\hline Serum lipase & $59(13-737)$ & $137(14-624)$ & $747(268-1191)$ & $674(84-2057)$ & 0.001 \\
\hline Serum amylase & $43(8-394)$ & $58.5(15-254)$ & $237(72-462)$ & $249(40-1107)$ & 0.006 \\
\hline \multicolumn{6}{|l|}{ Associated injuries } \\
\hline Head & $16(47.1 \%)$ & $13(46.4 \%)$ & $1(25.0 \%)$ & $0(0.0 \%)$ & 0.19 \\
\hline Thoracic & $21(61.8 \%)$ & $13(46.4 \%)$ & $3(75.0 \%)$ & $3(60.0 \%)$ & 0.54 \\
\hline Intraabdominal & $26(76.5 \%)$ & $21(75.0 \%)$ & $3(75.0 \%)$ & $4(80.0 \%)$ & 0.99 \\
\hline Head AIS & $4.7 \pm 0.9$ & $4.0 \pm 0.9$ & $3.0 \pm 0.0$ & $0.0 \pm 0.0$ & 0.06 \\
\hline Chest AIS & $3.0 \pm 0.6$ & $2.8 \pm 0.8$ & $2.7 \pm 0.6$ & $3.0 \pm 0.0$ & 0.67 \\
\hline Abdomen AIS & $2.8 \pm 1.0$ & $2.9 \pm 0.9$ & $3.0 \pm 0.8$ & $3.6 \pm 0.9$ & 0.46 \\
\hline ISS & $31.3 \pm 15.4$ & $24.5 \pm 13.4$ & $19.0 \pm 8.9$ & $21.6 \pm 11.4$ & 0.12 \\
\hline Intubated ETT & $21(61.8 \%)$ & $19(67.9 \%)$ & $1(25.0 \%)$ & $3(60.0 \%)$ & 0.42 \\
\hline FAST (Positive) & $14(41.2 \%)$ & $13(46.4 \%)$ & $3(75.0 \%)$ & $4(80.0 \%)$ & 0.63 \\
\hline Exploratory & $15(44.1 \%)$ & $15(53.6 \%)$ & $2(50.0 \%)$ & $5(100 \%)$ & 0.13 \\
\hline \multicolumn{6}{|l|}{ Laparotomy } \\
\hline Hospital length of stay & $11(1-61)$ & $16.5(1-57)$ & $36.5(21-56)$ & $19(19-40)$ & 0.03 \\
\hline ICU length of stay & $5(1-39)$ & $10(1-20)$ & $12(1-30)$ & $7(1-16)$ & 0.88 \\
\hline Ventilatory days & $6(1-26)$ & $8(1-13)$ & $30(30-30)$ & $3(3-6)$ & 0.26 \\
\hline Blood Transfusion & $26(76.5 \%)$ & $20(71.4 \%)$ & $2(50.0 \%)$ & $3(60.0 \%)$ & 0.64 \\
\hline Blood units transfused & $9.5(2-26)$ & $6(1-32)$ & $16.5(1-32)$ & $10(10-16)$ & 0.63 \\
\hline Mortality & $14(41.2 \%)$ & $7(25.0 \%)$ & $1(25.0 \%)$ & $0(0.0 \%)$ & 0.21 \\
\hline
\end{tabular}


With regards to clinical parameters, 38\% of patients had a GCS score of $<9$ (Table 3). Patients with a GCS $<9$ had a significantly higher ISS score (Average 37 compared to 21.3 in patients with GCS $>9, \mathrm{p}=0.001$ ) and higher mortality.

\begin{tabular}{|l|l|l|l|}
\hline \multicolumn{2}{|l|}{ Table 3: pancreatic injury by Glasgow coma scale } \\
\cline { 1 - 3 } & $\begin{array}{l}\text { GCS } \\
(\mathbf{n}=44)\end{array}$ & $\begin{array}{l}\text { GCS } \\
(\mathbf{n = 2 7})\end{array}$ & P-Value \\
\hline Hemorrhagic shock & $12(27.3 \%)$ & $13(48.1 \%)$ & 0.07 \\
\hline Injury severity score & $21.3 \pm 12.1$ & $37.0 \pm 12.7$ & 0.001 \\
\hline Pancreatic injury grade I-II & $36(81.8 \%)$ & $26(96.3 \%)$ & $0.07 \quad$ for \\
\hline Pancreatic injury grade III-IV & $8(18.2 \%)$ & $1(3.7 \%)$ & all \\
\hline Exploratory Laparotomy & $25(56.8 \%)$ & $12(44.4 \%)$ & 0.31 \\
\hline Hospital length of stay & $17(1-61)$ & $9(1-57)$ & 0.04 \\
\hline ICU length of stay & $5.5(1-39)$ & $9(1-35)$ & 0.81 \\
\hline Ventilatory days & $8(1-30)$ & $7(1-25)$ & 0.58 \\
\hline In-hospital complications & & & \\
\hline Wound Infection & $9(20.5 \%)$ & $2(7.4 \%)$ & 0.14 \\
\hline Pneumonia & $6(13.6 \%)$ & $5(18.5 \%)$ & 0.58 \\
\hline Acute respiratory & $2(4.5 \%)$ & $3(11.1 \%)$ & 0.29 \\
\hline syndrome & $1(2.3 \%)$ & $0(0.0 \%)$ & 0.43 \\
\hline Sepsis & $4(9.1 \%)$ & $5(18.5 \%)$ & 0.24 \\
\hline Acute renal failure & $1(2.3 \%)$ & $2(7.4 \%)$ & 0.29 \\
\hline Multiorgan failure & $1(2.3 \%)$ & $1(3.7 \%)$ & 0.72 \\
\hline Coagulopathy & $18(66.7 \%)$ & 0.001 \\
\hline Mortality & & & \\
\hline
\end{tabular}

Table 4 shows pancreatic injury in patients with and without hemorrhagic shock. There were 27 patients with SBP < 90 at presentation (38\%). Patients with hemorrhagic shock had a significantly higher ISS and higher mortality. Pancreatic injury scales did not differ significantly in patients with and without shock

Table 4: pancreatic injury with and without shock

\begin{tabular}{|c|c|c|c|}
\hline & $\begin{array}{l}\text { No hemorrhagic shock } \\
(n=46)\end{array}$ & $\begin{array}{l}\text { Hemorrhagic Shock } \\
(\mathrm{n}=25)\end{array}$ & P-Value \\
\hline GCS $\geq 9$ & $32(69.9 \%)$ & $12(48.0 \%)$ & \multirow{2}{*}{$\begin{array}{l}0.07 \text { for } \\
\text { all }\end{array}$} \\
\hline GCS $<9$ & $14(30.4 \%)$ & $13(52.0 \%)$ & \\
\hline Injury severity score & $23.2 \pm 13.6$ & $34.6 \pm 13.2$ & 0.001 \\
\hline Pancreatic injury grade I-II & $40(87.0 \%)$ & $22(88.0 \%)$ & \multirow{2}{*}{$\begin{array}{l}0.90 \text { for } \\
\text { all }\end{array}$} \\
\hline Pancreatic injury grade & $6(13.0 \%)$ & $3(12.0 \%)$ & \\
\hline
\end{tabular}




\begin{tabular}{|l|ll|l|}
\hline III-IV & \multicolumn{2}{|l|}{} & \\
\hline Exploratory Laparotomy & $24(52.2 \%)$ & $13(52.0 \%)$ & 0.98 \\
\hline Hospital length of stay & $16(1-61)$ & $11(1-57)$ & 0.44 \\
\hline ICU length of stay & $5.5(1-39)$ & $8.5(1-29)$ & 0.55 \\
\hline Ventilatory days & $8(1-30)$ & $6(1-22)$ & 0.43 \\
\hline In-hospital complications & & $5(20.0 \%)$ & \\
\hline Wound Infection & $6(13.0 \%)$ & 0.43 \\
\hline Pneumonia & $4(8.7 \%)$ & 0.07 \\
\hline $\begin{array}{l}\text { Acute respiratory distress } \\
\text { syndrome }\end{array}$ & $5(10.9 \%)$ & $0(0.0 \%)$ & 0.08 \\
\hline Sepsis & & & 0.53 \\
\hline Acute renal failure & $3(10.9 \%)$ & 0.19 \\
\hline Multiorgan failure & $2(4.3 \%)$ & 0.29 \\
\hline Coagulopathy & $0(0.0 \%)$ & $0(0.0 \%)$ & 0.17 \\
\hline Mortality & $10(21.7 \%)$ & 0.02 \\
\hline
\end{tabular}

Table 5 shows the pancreatic injury based on the ISS. Around $73 \%$ of patients had an ISS of equal or more than 16 . All the mortality cases had ISS $\geq 16$.

\begin{tabular}{|c|c|c|c|}
\hline \multicolumn{4}{|c|}{ Table 5: pancreatic injury by severity of injury } \\
\hline & ISS <16 $(n=19)$ & ISS $\geq 16(n=52)$ & P-Value \\
\hline GCS $\geq 9$ & $2(10.5 \%)$ & $23(44.2 \%)$ & \multirow[t]{2}{*}{0.004 for all } \\
\hline GCS $<9$ & $17(89.5 \%)$ & $27(51.9 \%)$ & \\
\hline $\begin{array}{l}\text { Pancreatic injury grade } \\
\text { I-II }\end{array}$ & $15(78.9 \%)$ & $47(90.4 \%)$ & \multirow[t]{2}{*}{0.20 for all } \\
\hline $\begin{array}{l}\text { Pancreatic injury grade } \\
\text { III-IV }\end{array}$ & $4(21.1 \%)$ & $5(9.6 \%)$ & \\
\hline Hemorrhagic shock & $2(10.5 \%)$ & $23(44.2 \%)$ & 0.01 \\
\hline Exploratory Laparotomy & $10(52.6 \%)$ & $27(51.9 \%)$ & 0.95 \\
\hline Hospital length of stay & $19(1-56)$ & $13(1-61)$ & 0.45 \\
\hline ICU length of stay & $4(1-16)$ & $8.5(1-39)$ & 0.09 \\
\hline Ventilatory days & $3.5(1-8)$ & $8(1-30)$ & 0.17 \\
\hline \multicolumn{4}{|l|}{ In-hospital complications } \\
\hline Wound Infection & $4(21.1 \%)$ & $7(13.5 \%)$ & 0.43 \\
\hline Pneumonia & $0(0.0 \%)$ & $11(21.2 \%)$ & 0.07 \\
\hline $\begin{array}{l}\text { Acute respiratory distress } \\
\text { syndrome }\end{array}$ & $0(0.0 \%)$ & $5(9.6 \%)$ & 0.16 \\
\hline Sepsis & $1(5.3 \%)$ & $8(15.4 \%)$ & 0.25 \\
\hline Acute renal failure & $0(0.0 \%)$ & $3(5.8 \%)$ & 0.28 \\
\hline Multiorgan failure & $0(0.0 \%)$ & $2(3.8 \%)$ & 0.38 \\
\hline
\end{tabular}




\begin{tabular}{|l|l|l|l|}
\hline Coagulopathy & $0(0.0 \%)$ & $1(1.9 \%)$ & 0.54 \\
\hline Mortality & $0(0.0 \%)$ & $22(42.3 \%)$ & 0.001 \\
\hline
\end{tabular}

Management and outcomes based on the injury grade are given in Figure 2. A total 37 patients underwent exploratory laparotomy (7 patients for pancreatic injuries [2 grade III had distal pancreatectomy and 5 grade IV had repair and drainage] and 30 patients for associated intra-abdominal injuries. Also 2 patients had laparoscopic exploration and drainage; one of them had distal pancreatectomy. Non operative management was done for 32 patients (19 grade I and 12 grade II and 1 grade III).

Almost one third of patients succumbed to their injuries (22/71). Mortality was not related to the grade of pancreatic injuries, but to the level of GCS, ISS score and initial SBP. The majority of deaths were observed in patients with low grade pancreatic injuries due to associated brain injury and polytrauma. One patient had pancreatic specific mortality in Grade III (multiorgan failure) and no mortality was reported in patients with Grade IV injuries.

Figure2:

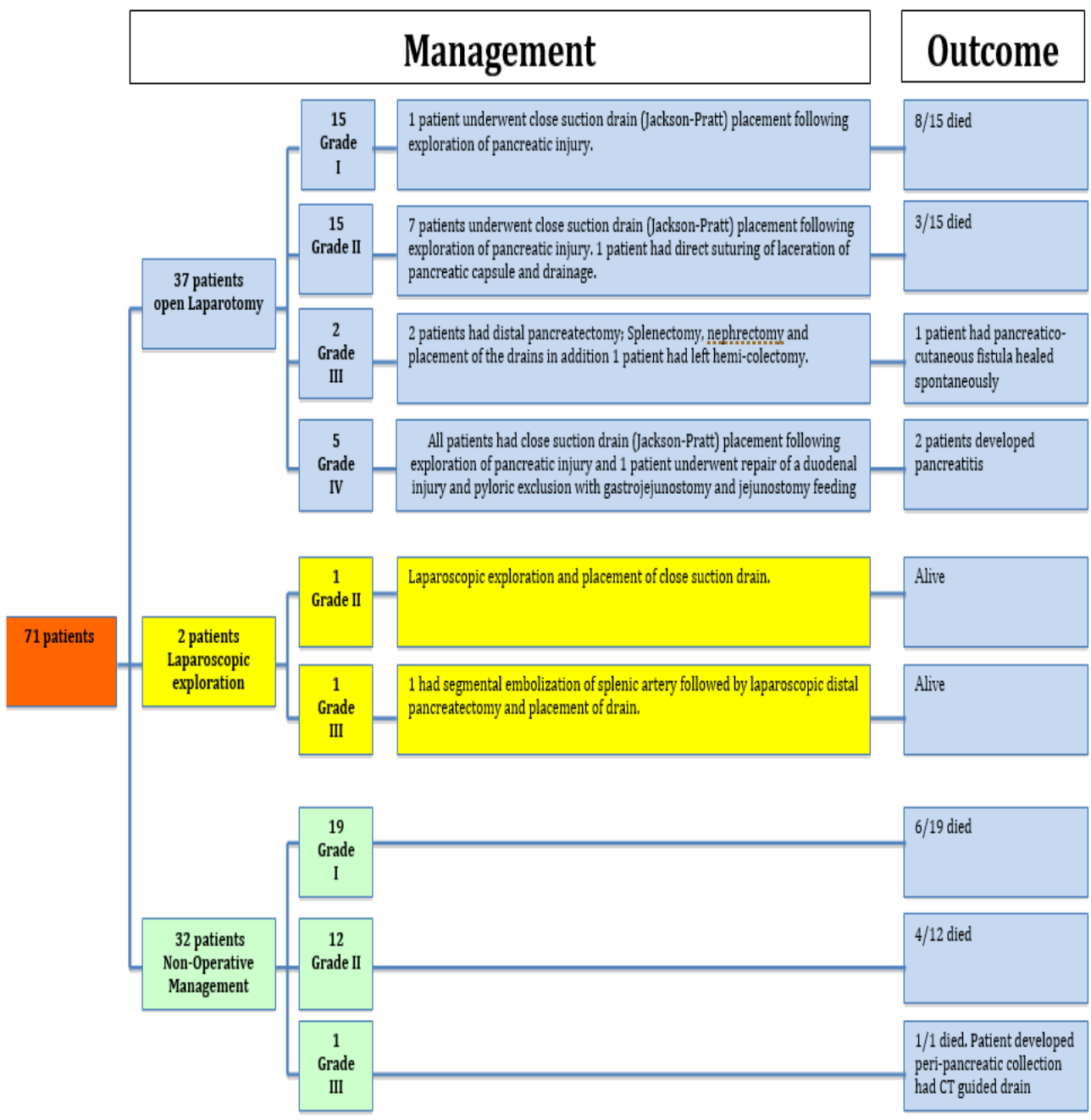




\section{Discussion}

Pancreatic injuries following abdominal trauma are uncommon. Radiologic and laboratory findings of acute pancreatic injury may be subtle however, the deep location in the retroperitoneal region makes its injury uncommon and its diagnosis more difficult. Accurate identification of pancreatic trauma, its grading, associated injury, and patient stability are mandatory to plan an appropriate treatment strategy. In this study, the prevalence of pancreatic trauma among total trauma admission and abdominal trauma were consistent with the concurrent literature. The blunt mechanism was the most common type of trauma with over half of them was following road traffic accidents and about a quarter was related to fall from height. These findings were similar to data recently published by Shibahashi et al., from Japan where blunt injuries predominated $(84.8 \%)$ with road traffic accidents $(57.7 \%)$, however, there was a sizable number of penetrating injuries due to attempted suicides (13.3\%) (4). Gupta et al., from India showed $42 \%$ sustained blunt injuries due to motorcycle accidents (12) , whereas Duchesne et al., reported $94.2 \%$ of patients who sustained blunt pancreatic injury due to motor vehicle collisions (24). These results are in stark contrast to data from South Africa reported by Krige et $\mathrm{Al}$., where over $70 \%$ of patients sustained penetrating injuries; the majority of them were due to gunshots (25).

Most of patients with pancreatic injuries were young males (average age of 30.8), similarly Gupta et al., and Siboni et al., reported injured patients with a median age of 24 $(12,26)$.

The mortality rate was high in the current study that was not reflecting the severity of pancreatic injury but was mainly related to the polytrauma, associated head injury and initial hemodynamics. Hwang et al., in their analysis of 75 patients showed that a GCS $<13$ was a significant predictor of mortality; $47.6 \%$ of their patients with a GCS $<13$ died (27).

The presence of hemorrhagic shock upon admission could be a predictor of mortality. Krige et al., and Hwang et al., had similar results, with mortality rate of $29 \%$ and $35.1 \%$ respectively in patients with a systolic blood pressure less than $90 \mathrm{mmHg}(25,27)$.

In this study, low grade (AAST I and II) pancreatic injuries accounted for nearly $90 \%$ of all injuries, similarly Siboniet al., reported $83 \%$ of patients with low grade injuries (26). Shibahashi et al., and Gupta et al., showed nearly $40-50 \%$ of their patients having low grade injuries, although individually, Gupta et al. had a higher number of grade III injuries $(4,12)$. Also, in a study by Krige et al., almost equal number of both low- and high-grade injuries was reported (25). Although multiple studies have shown a positive association between higher grade injuries and higher mortality rate, our study did not demonstrate significant association $(4,25,28)$. The location of injury was evenly distributed throughout the pancreas in the present study.

Pancreatic injuries are rarely isolated and are commonly associated with other visceral or extra abdominal injuries (29). In our study, isolated pancreatic injury was found in 8 patients (11.3\%), while Siboni et al., and Shibahashi et al., reported $20 \%$ and $29.6 \%$ isolated injuries respectively $(4,26)$. Traumatic head injury was the most commonly associated extra-abdominal injury in our cohort occurring in almost $43 \%$ of patients, followed by thoracic injuries in $27 \%$. Pancreatic injury is commonly associated with another solid organ injury with the spleen being the most common injured organ $(4,7,12,25,18$, 30).

In this study, there was an association between the grade of injury and elevation of pancreatic enzymes, with high grade injuries producing a significantly higher level of enzymes. Mahajan et al., in a prospective study of 164 patients concluded that both pancreatic enzymes showed $85 \%$ sensitivity and $100 \%$ specificity, although this was 
time-dependent, and was found to be significant when measured after 6 hours of injury (18). Although pancreatic enzymes are elevated in patients with intra-abdominal or craniofacial injuries (15), we observed that the degree of elevation was significantly proportional to the grade of pancreatic injury. However, this finding needs further support and explanation in prospective and larger studies.

FAST scan has a limited role in detecting solid organ injuries and with respect to the pancreas; the standard protocol of the examination does not cover its anatomical region $(9,31-32)$.

Abdominal CT scans are the diagnostic modality of choice for pancreatic injury in patients with blunt abdominal trauma who are hemodynamically stable, with a wide sensitivity range from 47-79\%. However, for detecting pancreatic duct injuries using multidetector CT scan; sensitivity reached $91-95 \%$ with specificity reaching 100\% (11). Generally, pancreas may appear normal in $20-40 \%$ of the acute blunt injuries if imaging is done in the early 12-hour post-trauma (17).

Patients with low grade pancreatic injuries are more likely to be managed non-operatively. Hemodynamic instability, which is the key factor in deciding to proceed with operative intervention, did not show any significant impact on the choice of management of pancreatic injury. The recent World Society of Emergency Surgery guidelines recommend NOM for hemodynamically stable grade I and selected grade II pancreatic injuries (9). In high level trauma center, NOM may be considered in selected hemodynamically stable patients with grade III pancreatic injuries who have proximal pancreatic body injuries without other abdominal injuries requiring surgery. NOM of Grade IV injury is controversial and should only be attempted in highly specialized centers with adequate availability of high-quality intensive care facilities as well as endoscopy and interventional radiology teams $(9,20,33)$. Shibahashi et al. had similar findings in their analysis of 743 patients where just over half of patients underwent a laparotomy with higher grade associated with an increased need for operative intervention, however they showed no superiority of operative management over NOM (4). In the study by Krige et al., all patients $(n=422)$ underwent a laparotomy and the majority of them requiring only pancreatic drainage (mostly grade I and II pancreatic injuries), 111 patients had a distal pancreatectomy and 19 patients had a pancreaticoduodenectomy (25). Although NOM is a feasible option in most cases of low grade pancreatic injuries, the failure of this conservative approach requiring subsequent surgery or a delayed surgical intervention due to an initially missed main pancreatic duct injury resulted in higher pancreas-specific mortality as well as morbidity. (34)

In the present study, only eight patients (11\%) had complications directly related to the pancreatic injury and $75 \%$ of these patients had high grade pancreatic injuries. Complications were more often in the higher-grade injury groups. Intra-abdominal collections were the most common complication, followed by pancreatitis which developed in two patients; one of them developed a pancreatico-cutaneous fistula. Both patients with pancreatitis had grade IV injuries. One patient with grade III injury developed pancreatic cyst. In the study by Al-Ahmadi et al., 38\% of all patients developed non-endocrine complications and were more common in patients with blunt injury as opposed to penetrating trauma (30). Gupta et al., had a complication rate of around $43 \%$ with most patients developing collections followed by pancreatitis (12). Krige et al., had a pancreas related complication rate of almost $21 \%$ with a similar picture of more complications with advancing grade of injury (25). They also noted that factors such as age, presence of shock, the need for a transfusion and the volume of blood transfused, damage control surgery, the need for a relook laparotomy and an associated vascular injury were significant predictors of complications.

Limitations: Our study is a retrospective, which is one of the limitations. Although the sample size is relatively small, but it is representative of the country population as the data were abstracted from the nationwide trauma database in Qatar. This trauma registry 
has regular internal and external validation as it is linked to the national trauma database bank (NTBD) in the USA. This study explores for the first time in our country the local experience in the diagnosis and management of traumatic pancreatic injury as this will improve our learning curve in this field. Our trauma center is the only level 1 tertiary trauma center in the country; it manages the moderate to severe cases free of charge for all the residents. Of note, the long-term outcomes are lacking in this study. Larger prospective studies are warranted for better early risk assessment and management.

\section{Conclusions}

Pancreatic injuries following blunt trauma are rare and the injured subjects are usually young male. However, most injuries are of low-grade severity. This study shows that shock, higher ISS and lower GCS are associated with worse in-hospital outcomes. Non-operative management may suffice in patients with lower grade injuries, which may not be the case in patients with higher grade injuries unless carefully selected. Larger prospective multicenter studies are still needed.

Author Contributions: “Conceptualization, HA.,AR,AH,GS and AE.; methodology, HA.,AH, and AE.; formal analysis, AE.; investigation, HA,AR.; data curation, HA,AH,AR.; writing-original draft preparation, HA.,AR,AH,GS and AE.; writing-review and editing, AE.; All authors have read and agreed to the published version of the manuscript.

Funding: "This research received no external funding".

Institutional Review Board Statement: "This retrospective study granted ethical approval from the medical research center and institutional review board of Hamad Medical Corporation, Doha, Qatar (IRB\#14409/14\& IRB \# MRC-01-18-003).

Informed Consent Statement: “Not applicable.”.

Data Availability Statement: Not applicable.

Acknowledgments: we thank trauma registry staff at HMC for their cooperation.

Conflicts of Interest: “The authors declare no conflict of interest.".

\section{References}

1. Akhrass R, Yaffe MB, Brandt CP, Reigle M, Fallon WF Jr, Malangoni MA. Pancreatic trauma: a ten-year multi-institutional experience. Am Surg. 1997. 63(7):598-604.

2. Larsen JW, Søreide K. The worldwide variation in epidemiology of pancreatic injuries. Injury. 2019 50(11): 1787-1789

3. Marin RS, Meredith JW. Management of acute trauma: Sabiston Textbook of Surgery: The Biological Basis of Modern Surgical Practice. Elsevier-Saunders. 2012; 430-470.

4. Shibahashi K, Sugiyama K, Kuwahara Y, Ishida T, Okura Y, Hamabe Y. Epidemiological state, predictive model for mortality, and optimal management strategy for pancreatic injury: a multicentre nationwide cohort study. Injury, 2020; 51:59-65

5. Jones RC. Management of pancreatic trauma. Am J Surg. 1985. 150:698-704.

6. Cimbanassi S, Chiara O, Leppaniemi A, Henry S, Scalia T. M, Shanmugaratnam K et al. Nonoperative management of abdominal solid-organ injuries following blunt trauma in adults. J Trauma Acute Care Surg. 2018. 84(3):517-531

7. Stawicki SP, Schwab W. Pancreatic Trauma: Demographics, Diagnosis, and Management. The American surgeon. 2009. 74(12):1133-45

8. Moore EE, Cogbill TH, Malangoni MA, Jurkovich GJ, Champion HR, Gennarelli TA, McAninch JW, Pachter HL, Shackford SR, Trafton PG. Organ injury scaling, II: Pancreas, duodenum, small bowel, colon, and rectum. J Trauma. 1990. 30(11):1427-9.

9. Ciccolini F, Kobayashi L, Kluger Y. et al. Duodeno-pancreatic and extrahepatic biliary tree trauma: WSES-AAST guidelines. World J Emerg Surg 2019. 14:56

10. Rickard MJFX, Brohi K, Bautz PC. Pancreatic and duodenal injuries: keep it simple. ANZ J Surg. 2005. 75:581-6.

11. Ho VP, Patel NJ, Bokhari F, et al. Management of adult pancreatic injuries: A practice management guideline from the Eastern Association for the Surgery of Trauma. J Trauma Acute Care Surg. 2017;82(1):185-199

12. Gupta A, Kumar S, Yadav SK, Mishra B Singhal M, Kumar A, Garg P. Magnitude, Severity, and Outcome of Traumatic Pancreatic Injury at a Level I Trauma Center in India. Indian J Surg. 2017. 79(6):515-520 
13. Elbanna KY, Mohammed MF, Huang S-C, Mak D, Dawe JP, Joos E, et al. Delayed manifestations of abdominal trauma: follow-up abdominopelvic CT in posttraumatic patients. Abdom Radiol. 2018. 43:1642-55.

14. Stewart BT, Sandstrom CK, O'Keefe G, Balmadrid B, Johnson GE. Multidisciplinary diagnosis and management of pancreatic trauma. Dig Dis Interv. 2018. 02:179-92.

15. Rau CS, Wu SC, Chien PC, Kuo PJ, Chen YC, Hsieh HY, et al. Identification of pancreatic injury in patients with elevated amylase or lipase level using a decision tree classifier: a cross-sectional retrospective analysis in a level I trauma center. Int J Environ Res Public Health. 2018. 15:277.

16. Linsenmaier U, Wirth S, Reiser M, Körner M. Diagnosis and classification of pancreatic and duodenal injuries in emergency radiology. Radiographics. 2008. 28:1591-602.

17. Kumar A, Panda A, Gamanagatti S. Blunt pancreatic trauma: a persistent diagnostic conundrum? World J Radiol. 2016. 8:159-73.

18. Mahajan A, Kadavigere R, Sripathi S, Rodrigues GS, Rao VR, Koteshwar P. Utility of serum pancreatic enzyme levels in diagnosing blunt trauma to the pancreas: a prospective study with systematic review. Injury. 2014. 45:1384-93.

19. Moore EE, Cogbill TH, Malangoni MA, Jurkovich GJ, Champion HR, Gennarelli TA, McAninch JW, Pachter HL, Shackford SR, Trafton PG. Organ injury scaling, II: Pancreas, duodenum, small bowel, colon, and rectum. J Trauma. 1990. 30(11):1427-9.

20. Biffl WL, Moore EE, Croce M, Davis JW, Coimbra R, Karmy-Jones R, McIntyre RC Jr,Moore FA, Sperry J,Malhotra A, et al.Western Trauma Association critical decisions in trauma: management of pancreatic injuries. J Trauma Acute Care Surg. 2013. 75:941-946.

21. Girard E, Abba J, Cristiano N, Siebert M, Barbois S, Létoublon C, Arvieux C. Management of splenic and pancreatic trauma. J Visc Surg. 2016. 153:45-60.

22. Girard E, Abba J, Arvieux C, Trilling B, Sage PY, Mougin N, Perou S, Lavagne P, Létoublon C. Management of pancreatic trauma. J Visc Surg. 2016. 153:259-268.

23. Koganti SB, Kongara R, Boddepalli S, Mohammad NS, Thumma V, Nagari B, Sastry RA. Predictors of successful non-operative management of grade III \& IV blunt pancreatic trauma. Ann Med Surg. 2016. 10:103-109.

24. Duchesne JC, Schmieg R, Islam S, Olivier J, McSwain N (2008) Selective nonoperative management of low-grade blunt pancreatic injury: are we there yet? J Trauma. 65:49-53

25. Krige JE, Kotze UK, Setshedi M, Nicol AJ, Navsaria PH. Prognostic factors, morbidity and mortality in pancreatic trauma: A critical appraisal of 432 consecutive patients treated at a Level 1 Trauma Centre. Injury. 2015. 46(5):830-6

26. Siboni S, Kwon E, Benjamin E, Inaba K, Demetriades D. Isolated blunt pancreatic trauma: A benign injury? J Trauma Acute Care Surg. 2016. 81(5):855-859

27. Hwang SY, Choi YC. Prognostic determinants in patients with traumatic pancreatic injuries. J Korean Med Sci. 2008. 23: 126-130.

28. Antonucci N, Di Saverio S, Caroni V, Biscardi A, Giugni A, Cancellieri F, Tugnoli G. Prognosis and treatment of pancreaticoduodenal traumatic injuries: which factors are predictors of outcome? Journal of Hepato-Biliary-Pancreatic Sciences. 2010. 18(2): 195-201.

29. Sims EH, Mandal AK, Shalter T, Flemming AW, Lou MA. Factor affecting outcome in pancreatic trauma. J Trauma. 1984; 24:125-128

30. Al-Ahmadi K, Ahmed N. Outcomes after pancreatic trauma: experience at a single institution. Can J Surg. 2008. 51(2): 118-124.

31. Körner M, Krötz, MM, Degenhart C, Pfeifer KJ, Reiser MF, Linsenmaier U. Current Role of Emergency US in Patients with Major Trauma. RadioGraphics 2008. 28(1); 225-242.

32. Sato M, Yoshii H. Reevaluation of Ultrasonography for Solid-Organ Injury in Blunt Abdominal Trauma. Journal of Ultrasound in Medicine. 2004. 23(12); 1583-1596.

33. Bhasin DK, Rana SS, Rawal P. Endoscopic retrograde pancreatography in pancreatic trauma: need to break the mental barrier. J Gastroenterol Hepatol. 2009. 24(5):720-728.

34. Haulik L, Tóth B, Issekutz A, Gartner B. Pancreatic injury in blunt abdominal trauma: early versus late diagnosis and surgical management. Magy Seb. 2001 Oct; 54(5):309-13. 